
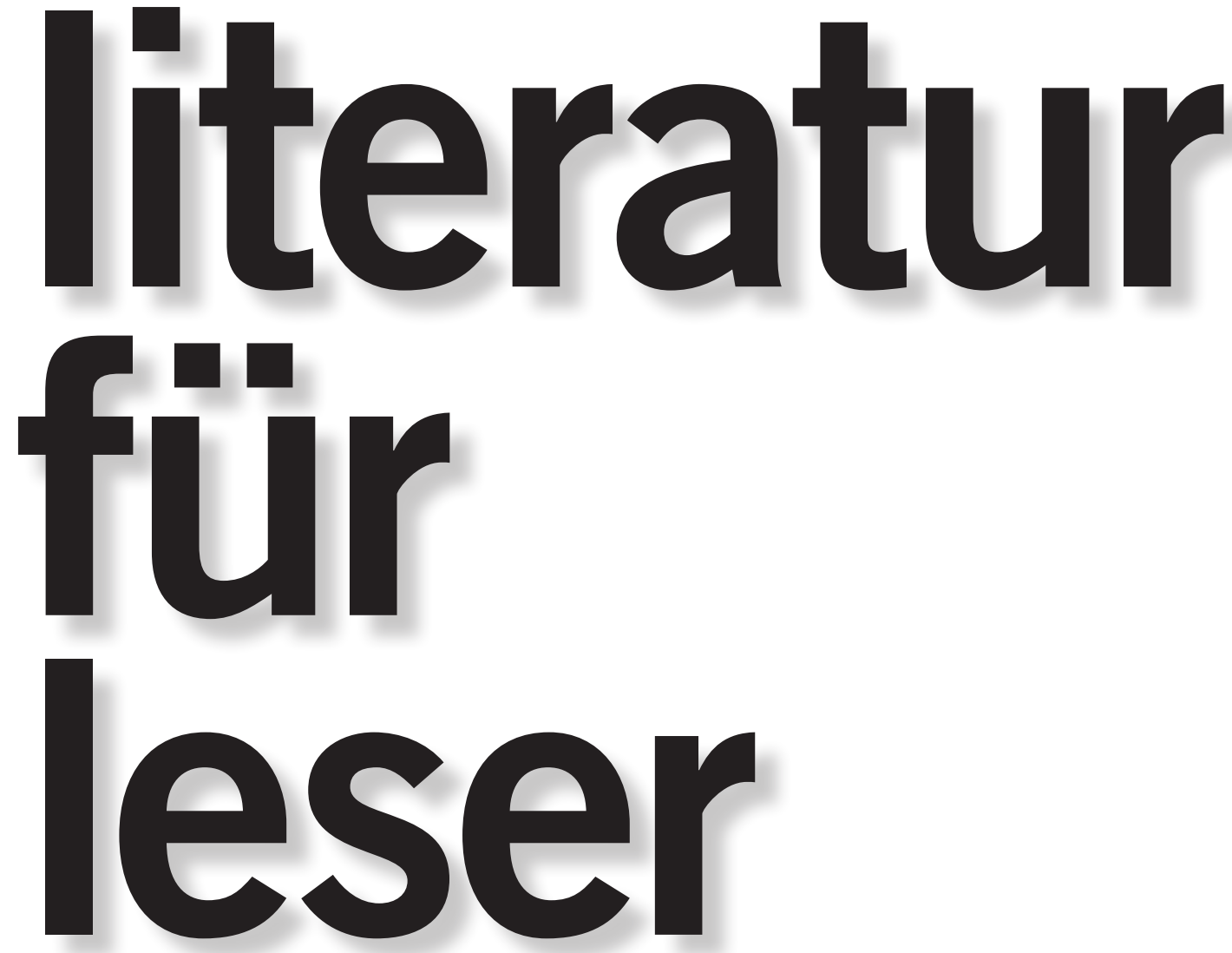
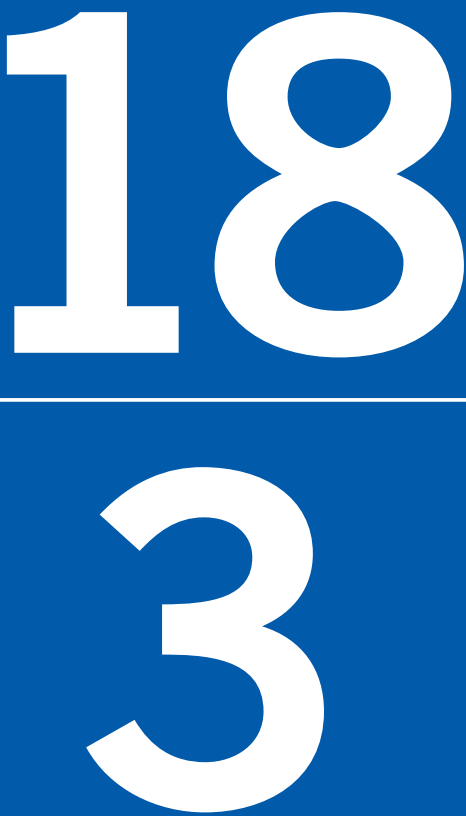

Georg Heyms nachgelassene Prosa und Schriften

Herausgegeben von Lars Amann

Mit Beiträgen von Frank Krause, Andreas Kramer, Roland Innerhofer, Moritz Baßler, Katharina Scheerer, Wolfgang Braungart und Barbara Neymeyr 


\section{Getarnte Vitalität. Von Alfred Doves Caracosa (1894) zu Georg Heyms Bagrow (1911)}

Anfang der 1970er Jahre hatte Gunter Martens die vitalistischen Perspektiven des literarischen Werks von Georg Heym detailliert herausgearbeitet. Der Glaube, im gesteigerten Erleben der aktiven Erfüllung unabweisbarer Bedürfnisse offenbare sich der sinnstiftende Ursprung des Lebensprozesses, leitet Heyms Darstellungen spontaner Aufbrüche aus gefühlsarmer Sinnleere ebenso wie seine Diagnosen zerstörter Hoffnungen auf ein erfülltes Leben; noch Heyms Szenen der sinnlich faszinierenden Vernichtung lebensfeindlicher Stätten und seine paradoxen Motive einer vitalisierten Todesmacht, die eben jene berauschende Energie verausgabt, an denen es ihren Opfern mangelt, bewegen sich im Horizont vitalistischen Denkens. ${ }^{1}$

Die Problematik der getarnten Freisetzung von Vitalität ist in diesem Zusammenhang bislang vernachlässigt worden. Schon die Wertschätzung, die Heym dem Roman Caracosa (1894) von Alfred Dove zwischen 1904 und 1909 entgegenbringt, verdankt sich Sympathien mit dem widersprüchlichen Versuch, unter der Maske der Konformität mit sozialen Normen, die den Zugang zur Lebensfülle verlegen, auf verdeckte Weise ein bewegtes Leben zu führen. Noch 1912 fragt Heym, ob ein Genius, der aus dem Chaos schöpfe, verdeckt in einem Staat wirken könnte, dessen starre Ordnung ihm feindlich gesinnt sei; die Antwort fällt negativ aus, es "sei denn, daß" sich das Genie "genügend verstellen kann", doch der Widerspruch zwischen seinen Zielen und Mitteln bleibt auch dann bestehen, denn „die Mühen, die er darauf verwenden muß, nicht anders zu erscheinen, wie die allgemeine Horde, bedeuten eine so entsetzliche Vergeudung seiner Kräfte". ${ }^{2}$ Zwei bisher wenig beachtete, 1911 verfasste Prosatexte aus Heyms Nachlass arbeiten sich an der Krise von Projekten der getarnten Freisetzung von Vitalität ab und seien - als Beitrag zur Bestandsaufnahme der Probleme, die in Heyms späten Prosastücken und -skizzen ausgelotet werden im Folgenden eingehender dargestellt.

Das Verständnis des ersten Textes erfordert einen Exkurs zu Heyms Caracosa-Lektüre, die auch einen Einblick in die Geschichte seines Umgangs mit Problemen der getarnten Realisierung von Vitalität erlaubt (1.). Jene unbetitelte, in Teilen wieder verworfene Prosaskizze Heyms führt ein sinnentleertes Stadtleben vor Augen und stellt - unter Anspielung auf Doves Roman - die poetische Bejahung maskierter Vitalität als absurde Selbstentfremdung dar; Heym streicht die letztere Diagnose aber wieder durch und überbietet sie mit der Darstellung eines umfassenden Sinnentzugs: Der Erzähler, der im Bann sozialer Entfremdung den Lebenswillen eingebüßt hatte,

1 Vgl. Gunter Martens: Expressionismus und Vitalismus. Ein Beitrag zur Genese und Deutung expressionistischer Stilstrukturen und Motive. Stuttgart, Berlin, Köln u. Mainz 1971 (= Studien zur Poetik und Geschichte der Literatur), S. 180-257.

2 Georg Heym: Über Genie und Staat. In: Georg Heym. Dichtungen und Schriften. Bd. 2: Prosa und Dramen. Hrsg. v. Karl Ludwig Schneider. Hamburg u. München 1962, S. 174-176, hier S. 174. 
flieht aus Liebesmangel einsam in den unerfüllten Rausch (2.). In Heyms unvollendeter Erzählung Bagrow verliert der Versuch eines revolutionären Attentäters, im getarnten Handeln eines Mitverschwörers den Ausdruck genialer Vitalität zu erkennen, aus der Sicht eines umfassend verunsicherten Bewusstseins jede Grundlage (3.). Bis 1910 stellt Heym das Scheitern von Versuchen der getarnten Freisetzung von Vitalität dar, um den Lebenskult der Literatur der Jahrhundertwende zu komplizieren; im expressionistischen Spätwerk gilt getarnte Vitalität als idealisierendes Konstrukt, das in der entfremdeten zeitgenössischen Lebenspraxis erst gar nicht Fuß fassen kann (4.).

\section{Alfred Doves Caracosa in Heyms Schriften von 1904 bis 1909}

"Auf dem ersten Blatt des" von Heym „ab 1904 für Gedichtreinschriften benutzten Heftes steht"

\begin{tabular}{|c|c|c|c|c|c|c|}
\hline $\begin{array}{l}f, f \\
\text { Ogni }\end{array}$ & $\begin{array}{l}\text { cfc, } \\
\text { be }\end{array}$ & $\begin{array}{l}\text { cfc. } \\
\text {-ne }\end{array}$ & $\mathrm{BaB}$ & $\begin{array}{l}\mathrm{f}, \\
\mathrm{Ca}\end{array}$ & $\begin{array}{l}f, \\
-r a\end{array}$ & $\begin{array}{l}\mathrm{bfb}, \\
-\mathrm{co}\end{array}$ \\
\hline
\end{tabular}

Mit der ersten Seite dieses Heftes, das am 20. November begonnen und im Mai 1905 durch ein umfangreicheres ersetzt wurde, stellt Heym seine Lyrik zumindest punktuell unter ein vertontes Motto, das Doves Caracosa entnommen ist. ${ }^{4} \mathrm{Im}$ Tagebuch bezeichnet er diesen Roman am 17. Juli 1905 als eines seiner "Lieblingsbücher" und beschließt den Eintrag mit dem Ausruf: „Ognibene - - Caracosa!"5 Der Eintrag vom 25. Dezember 1906 belegt, dass er in den Hauptfiguren des Romans, die zum Leidwesen des männlichen Protagonisten nicht zueinander finden, sein eigenes unglückliches Lebensgefühl wiedererkennt; mit der musikalischen Evokation des Namenspaars will er eine tröstliche Stimmung heraufbeschwören:

\footnotetext{
Eine verwandte Stimmung, die mich wieder etwas beruhigt, finde ich nur in dem unglücklichen Leben Ognibenes und Caracosas. Wenn ich so wieder einmal ganz unglücklich bin, dann höre ich wieder die schmerzlichen Töne

$$
\text { - Ognibene - - - Caracosa - }
$$
}

und bleibe dann noch etwas vor dem Clavier sitzen und greife noch einige Akkorde. ${ }^{6}$

Am 15. September 1907 charakterisiert Heym mit der Zeile "Ognibene - Caracosa" den Anblick einer Frau, in die er unglücklich verliebt ist; sie trägt denselben Spitznamen wie die Viktoria auf der Berliner Siegessäule: „Goldelse gesehen. Wunderschön und dabei traurig". ${ }^{7}$ Von Mitte 1905 bis Ende 1909 zieht sich das Namenspaar leitmotivartig durchs Tagebuch; am 10. Oktober 1909 ruft es erneut die melancholische Schönheit unglücklicher Liebe hervor: „Ognibene! Caracosa! Wann wird wieder

3 Nina Schneider (Hrsg.): Georg Heym 1887-1912. Eine Ausstellung der Staats- und Universitätsbibliothek Carl von Ossietzky Hamburg. Berlin 1988, S. 21.

4 Zur Datierung siehe Nina Schneider (Hrsg.): Am Ufer des blauen Tags. Georg Heym. Sein Leben in Bildern und Selbstzeugnissen. Glinde 2000, S. 213.

5 Georg Heym. Dichtungen und Schriften. Bd. 3: Tagebücher Träume Briefe. Hrsg. v. Karl Ludwig Schneider. München 1986, S. 33.

6 Ebd., S. 77-78.

7 Ebd., S. 95. Siehe auch den Eintrag vom 6.11.1906 in: Ebd., S. 74.

\section{I literatur für leser 3/18}


einmal etwas ähnliches geschrieben werden. Ognibene. Caracosa. Wen von beiden liebe ich mehr."8 Am 27. Dezember 1909 bezieht er den Namen des Autors in die eingespielte Beschwörung der Stimmung ein, die der Roman bei ihm auslöst: „Dove - Ognibene - Caracosa", und er ,folgert' aus der Reihe dieser und weiterer Namen, dass es nicht möglich sei, glücklich zu sein. ${ }^{9}$

Mit dem sangbaren Namenspaar bezieht sich Heym auf das Kirchenglockengeläut am Ende von Doves Roman, das dem Mönch Salimbene wie eine Anrufung von Ognibene und Caracosa anmutet. Ognibene hatte sich aus enttäuschter Liebe zu Caracosa den Franziskanern angeschlossen und den Namen Fra Salimbene angenommen; seine unerwiderte Liebe zu Caracosa überdauert deren Tod, und das Festgeläut, das der sterbende Salimbene hört, wird ihm zur Beschwörung ihrer Zusammengehörigkeit. Dass der Tod Salimbenes auf den ersten Weihnachtstag fällt, ist doppelt bedeutsam: Ognibene hatte sich dem Orden aus weltlichen Gründen angeschlossen, und Caracosa war am ersten Weihnachtstag geboren worden. Salimbenes christlicher Dienst am Erlöser, dessen Geburt an diesem Tag gefeiert wird, gilt in Wahrheit der verdeckten Bewältigung enttäuschter Liebe; als Caracosa noch lebte, hatte Salimbene mit seiner Weltflucht immer auch deren Seelenruhe zu erschüttern gesucht. Die vorgebliche Entsagung als Mönch diente inm als verzweifelter Appell an die Geliebte, deren Nähe er auch als Ordensmitglied suchte, ihm ihre Aufmerksamkeit zu schenken.

Dass die Glocken, die das Namenspaar evozieren, zunächst „freud- und leidlos" schallen, ${ }^{10}$ verweist auf die Avitalität des Klosterdienstes, doch am Ende steigert sich ihr Klang zu einem Fest, das im schönen Schein Trost spendet. Praktisch ist Salimbenes Lebensweg der Tarnung seiner Liebe längst gescheitert: Caracosa starb beim Sturz von ihrem Pferd, als sie sich einem Zug von Flagellanten in den Weg stellte, die von Salimbene angeführt wurden. Zuvor hatte sie deren Askese noch als unchristlichen Frevel am Leib als Tempel des Heiligen Geistes gebrandmarkt. Die tragische Szene unterstreicht die Vergeblichkeit eines Liebeswerbens durch Selbstgeißelung, doch der Schluss des Romans schönt Salimbenes unerschütterliches Festhalten an der Liebe zu Caracosa auf und bejaht auf diese Weise auch dessen Lebensweg als Ausdruck vitaler Energien.

In seinem Tagebuch notiert Heym, dass er beide Hauptfiguren gleichermaßen liebt, was auf eine Identifikation mit Ognibene schließen lässt, denn nur aus dessen Sicht sind beide Figuren gleichermaßen positiv. Ognibenes Vater Guido di Adamo hatte der früh verwaisten Caracosa Schutz vor den religiösen Unruhen in ihrer Heimat gewährt; so wächst sie in der Fremde auf, wo sie ihren ausgeprägten Sinn für Freiheit kultivieren kann. Ognibene fühlt sich derweil in den Erwartungen gefangen, die sein Vater an ihn heranträgt, und findet zu keiner Rolle, in der er Caracosa, die wie eine Stiefschwester mit inm heranwächst, selbstsicher und attraktiv erscheinen könnte. Ognibene und Caracosa stellen komplementäre Spielarten des Scheiterns eines freien passionierten Lebens dar; während Caracosa sich unter Verzicht auf Nähe mit Stolz und kluger Anpassung von einem Leben in Unfreiheit distanziert, sucht Ognibene vergeblich nach Erfüllung in der bedingungslosen Hingabe an und Anerkennung

8 Ebd., S. 132.

9 Ebd., S. 133.

10 Alfred Dove: Caracosa. Historischer Roman aus dem dreizehnten Jahrhundert. Bd. 2. Stuttgart 1894, S. 380. 
durch seine geliebte Caracosa und gibt seinen Anspruch auf weltliche Freiheiten auf. Ognibenes verzweifelte Bedürftigkeit lässt die attraktiv selbstsichere, umsichtig auf Abstand bedachte Caracosa indessen kalt. Nachdem der Versuch, seine soziale Entfremdung in der Liebe zu Caracosa zu überwinden, umfassend gescheitert ist, flieht er - in trotzigem Gegensatz zur Religiosität seines Vaters - in die Rolle eines Mönchs:

\footnotetext{
Von Stund an gewann sein Betragen eine merkwürdige Sicherheit. Er trat so ruhig auf, wie ein Verschworener, der für den Notfall das befreiende Gift bequem erreichbar bei sich führt. Gab ihm der Vater dann und wann ein unwirsches Wort, wozu sich jedoch nur selten Anlaß fand, kam das Familiengespräch gelegentlich auf Orlando, erschien inm Caracosa einmal besonders reizend, übermäßig kalt, oder auch gar zu herablassend - so griff er gleichsam in Gedanken nach dem rettenden Saft, um sich von dessen unversehrter Gegenwart im stillen tröstlich zu überzeugen: er sah sich heimlich auf der Flucht, nur wenige Straßen weit; er hörte die Thür von San Francesco del Prato dumpf hinter sich zuschlagen. ${ }^{11}$
}

Ognibene wird aus „weltlichen Beweggründen und nicht ohne Hintergedanken des Ehrgeizes" Mönch. ${ }^{12}$ Sein gesteigertes religiöses Empfinden im Vorfeld der Flucht in den Orden ist sinnlich-poetisch fundiert:

\footnotetext{
Noch nie zuvor hatte inn der Steinodem des weiten Gotteshauses so schaurig überhaucht; noch nie war ihm der Harzduft des Weihrauchs, das Auf- und Abwogen des Chorgesangs, der zitternde Schein der Kerzen, deren tropfendes Wachs die Leuchter fein erklingen ließ, so stark auf die Nerven gefallen, wie heut; noch niemals hatte er mit so träumerischer Andacht über die mächtige Treppe in die Wölbung der Nische hinterm Hochaltar hinaufgeblickt. Welch ein inbrünstiges Verlangen ward in ihm rege nach der Freundlichkeit und Leutseligkeit Gottes, unseres Heilandes, von der die Predigt handelte, nach dem Bade der Wiedergeburt, wodurch er uns nach seiner Barmherzigkeit selig machen will!13
}

Als Mönch kultiviert Ognibene sinnliche Freuden an gutem Essen und Wein und seine Wertschätzung der Gelehrsamkeit und Beredsamkeit, und als Ordensbruder mit respektabler Karriere sucht er erneut - und wiederum vergeblich - die Nähe zu Caracosa. Ognibene nutzt eine soziale Rolle, deren religiöses Ethos ihm fremd ist, dazu, seinem Vater zu trotzen und den Spielraum für sinnliche Genüsse, die glanzvolle Entwicklung intellektueller Fähigkeiten und die Erschütterung von Caracosas Seelenruhe durch ostentative Weltentsagung aus enttäuschter Liebe zu wahren und zu erweitern. So rettet er sich in eine Lebensform, in der er in getarnter Form gelegentlich seiner Sinnlichkeit und gedanklichen Unabhängigkeit frönt und seinem praktisch vorerst gescheiterten Lebensziel einer Liebesverbindung mit Caracosa auf verkappte Weise verpflichtet bleibt. Der Versuch, seine Vitalität in der Tarnung der Askese zu behaupten, mündet in unaufgelöste Selbstwidersprüche, doch das Glockenmotiv zeigt an, dass der Konflikt zwischen Anspruch und Wirklichkeit die Energien, aus denen sich der eingeschlagene Lebensweg speist, nicht entwerten kann.

\section{2. ,Ognibene - Caracosa‘ in der expressionistischen Groteske}

Doves punktuelle Aufschönung des Leidens an versagter Lebensfülle, auf die Heym mit der Beschwörung der Namen von Ognibene und Caracosa anspielt, passt zum literarischen Jugendstil, der die Hingabe an die berauschende Schönheit von Artefakten als Manifestation eines natürlichen, sinnlich-erotisch ausgerichteten Willens

11 Dove: Caracosa. Bd. 1, S. 325.

12 Ebd., S. 340-341.

13 Ebd., S. 328-329. 
zum Leben feiert; auch dort, wo dieser Wille nur in gebrochener Form zum Ausdruck gelangen kann, wird er in poetisch aufgeschönter Form - aller Vergeblichkeit zum Trotz - bejaht. ${ }^{14}$ Gewiss, Dove kompliziert diese Haltung, denn der Jugendstil feiert die Entsagung nicht als ein verborgenes ,Ja' zum Leben, sondern stellt sie kritisch als Ausdruck einer verdrängten Sinnlichkeit dar. Der Jugendstil ästhetisiert den kirchlichen Raum aus säkularer Distanz, während die ästhetischen und christlichen Perspektiven in Salimbenes Konversionserlebnis verschmelzen; die Askese bewahrt sein Begehren um den Preis einer vorläufigen Entsagung gleichsam auf. ${ }^{15}$ Der Erzähler registriert die ästhetisch-religiöse Sensibilität seines Protagonisten und deren Verbindung mit sinnlicher Vitalität aus psychologischer Distanz und führt eine Erschöpfung einschlägiger Kräfte vor Augen, die hinter die Ansprüche des Jugendstils auf sinnliche Fülle zurückfällt, kündigt ihm aber nicht die Sympathie auf. Die Flagellantenszene des Romans, in der die religiöse Askese als pervertierte Sinnlichkeit entlarvt werden soll, setzt die Kritik des Jugendstils an leibfeindlichen Tendenzen im Christentum indessen fort. ${ }^{16}$

Auch Heym kompliziert in seinen frühen Schriften den Lebenskult der Jahrhundertwende, ohne ihm die Sympathie zu verwehren; so stellt er unversöhnliche Widersprüche zwischen enthusiastischer Neigung und versagter Fülle in ihrer Untröstlichkeit dar, ${ }^{17}$ doch noch im Dezember 1909 arbeitet er am Versuch zu einem jugendstilhaften Ritus, ${ }^{18}$ und mit der rituellen Beschwörung des Ausklangs von Doves Roman bejaht er nach wie vor die poetische Tröstung über Salimbenes Ausharren in seinem vergeblichen Liebeswunsch. In einer nachgelassenen kurzen Prosaskizze aus dem Jahre 1911 verwendet Heym das ,Ognibene - Caracosa'-Motiv hingegen in grotesker Verfremdung. Seine anspruchsvoller gewordene, expressionistisch ausgerichtete Suche nach metaphysischem Sinn in ungekränkten Werterlebnissen kann sich über die Durchkreuzung unveräußerlichen Lebenssinns nicht länger im poetischen Schein hinwegtrösten: ${ }^{19}$

Ich beschloß mich verbrennen zu lassen, denn die ganze Straße war voll Geschrei. Und das Gelb der Kranken, deren riesige Backen aus allen Fenstern hingen, wollte ein Opfer. Wahrhaftig, sie sahen aus, wie riesige große Affen. Und ihre Maultaschen bedeckten drei Stockwerke. Wenn sie sich begrüßten, so schlugen sie ihre Stirn gegeneinander. Und die riesige Elephantiasis ihrer Backen bedeckte die Straße unten wie mit schwarzer Nacht. Wenn man unten stand, so hätte man ihre gewaltigen Ohrlappen für riesige fliegende Hunde halten können, die um den Mond stürmten mit einem Geschrei, das wie das Auskratzen von Töpfen klang.

[Aber, wo die Straße zuende war, waren die grünen Schilfriesen, blaugrün, und man konnte sie nicht ansehen, ohne geblendet zu werden. Ein Mann ging hindurch. Sein Kopf war eine riesige Soldatenflöte, die in seiner Luftröhre stak. Und wenn er atmete, sang die Flöte. Und sie sang: Ognibene - Caracosa. ${ }^{20}$

14 Vgl. Frank Krause: Literarischer Expressionismus. Göttingen 2015, S. 31.

15 Die Stilisierung des kirchlichen Raumes zum Ort der sinnlichen Offenbarung erneuernder Naturkräfte findet sich unter eindeutig säkularen Vorzeichen beim Jugendstil-Autor Max Dauthendey; siehe dazu Dominik Jost: "Jugendstil und Expressionismus". In: Expressionismus als Literatur. Gesammelte Studien. Hrsg. von Wolfgang Rothe. Bern u. München 1969, S. 87-106, hier S. 93-98.

16 Bernd W. Seiler nimmt an, dass die Flagellantenmotivik in Heyms zwischen 1905 und 1908 verfasstem Drama Arnold von Brescia von Doves Roman angeregt wurde (vgl. Bernd W. Seiler: Die historischen Dichtungen Georg Heyms. Analyse und Kommentar. München 1972, S. 233). Auch hier erscheint die Geißelung als pervertierte Spielart der Sinnlichkeit (Georg Heym: Arnold von Brescia. Trauerspiel in fünf Akten. In: Georg Heym. Bd. 2, S. 417-546, hier S. 487-488).

17 Vgl. Martens: Expressionismus und Vitalismus, S. 182-188.

18 Georg Heym: Versuch einer neuen Religion. In: Georg Heym. Bd. 2, S. 164-172.

19 Zu den Erlösungsvorstellungen des expressionistischen Vitalismus siehe Krause: Expressionismus, S. 110-129.

20 Georg Heym: Skizzen. In: Georg Heym. Bd. 2, S. 157-161, hier S. 158. 
Die Skizze entwirft zunächst grotesk deformierte menschliche Körper, deren Qualitäten und Umgebungen bedeutsam kontrastieren. Die verdunkelte Stadt ist durch abstoßendes Geschrei einer Gemeinschaft kränklich gelber, übergroßer Personen gekennzeichnet, die im blendenden Blau-Grün erstrahlende, übergroße Natur durch die Flötenmusik eines einzelnen Mannes. Die Farbe der degenerierten Köpfe bringt den lebensfeindlichen Wirkungsbereich einer Opfer erheischenden, mythisch anmutenden Macht zum Ausdruck, während der künstliche Flöten-Kopf auf die Idee einer Versöhnung von Schönheit und Traurigkeit angesichts unglücklicher Liebe anspielt, die sich aber weder in der hässlichen Stadt noch im Bild einer übergroßen Querflöte retten lässt, die anstelle des Kopfes aufrecht in der offenen Luftröhre steckt. Der Flöten-Mann mag sich um eine Mimikry an die umgebende Schilf-Natur bemühen, doch sein verstörender Anblick bricht jede tröstende Illusion ihres Gelingens. Heym streicht die Passage über das Leben hinter dem Straßenende jedoch wieder durch und führt den Entschluss des Erzählers, sich verbrennen zu lassen, auf die Rede eines Betrunkenen zurück, der vereinsamt mit Bäumen und Dingen spricht und seinen Rausch als Flucht vor dem Gefühl rechtfertigt, nicht geliebt zu werden.

Die Streichung des ,Ognibene - Caracosa'-Motivs mag der Rücksicht auf das Lesepublikum geschuldet sein: zwar sind die Motive jener beiden Absätze wie in einem Prosagedicht zu einem stimmigen Kontrast gefügt, doch die Anspielung auf Doves Roman bliebe für die meisten Leser obskur. ${ }^{21}$ Die Kritik des Versuchs, aus Mangel an Liebe in poetisch illusionierte Weltbezüge zu fliehen, wird stattdessen aus der Sicht eines Betrunkenen entwickelt, der seiner avitalen Verfassung auch im Rausch nicht entgeht: Seine entgrenzte Wahrnehmung ist nicht ansprechend, das Blanke und Klare dessen, was er sieht, bildet einen beklagenswerten Kontrast zur eigenen Verfassung, und im Gespräch mit den stummen Dingen bleibt er allein und wünscht sich, die Zeit würde stillstehen:

\footnotetext{
Hört ihr, wie der Wind pfeift. Ihr habt eine kalte Faust, Wind. Ihr setzt rote Nasen auf.

Kurzum, habe ich nicht recht Herr Mond, Herr gelber Puffärmel, Herr Laterne, Herr Einauge.

Ihr seid auf den Tisch geworfen, wie ein Eierkuchen.

Ach Ihr, Ihr bleibt immer blank, immer klar. Ihr waret vielleicht schon einmal grau, wie ein Streusandglas voller Jahre.

Ach ich bin hier ganz allein. Warum darf es überhaupt Nacht werden. Mindestens sollte die Zeit bei Mitternacht aufhören. Denn was nachher kommt zwischen Mitternacht und erstem Hahnenschrei -

Würde mich ein Mädchen lieben, wäre ich nie so betrunken. Herr Ulme, Herr Marktbrunnen. ${ }^{22}$
}

Der gestrichene Befund, dass poetische Tröstungen im Geiste eines skeptisch gebrochenen Lebenskults der Jahrhundertwende in eine absurde Selbstentfremdung münden, wird mit der expressionistischen Zeitdiagnose überboten, dass sich der Vitalitätsverlust im mythisch anmutenden Bann eines fremdbestimmten Großstadtlebens weder in der Liebe noch im Rausch überwinden lässt. Im Kontext von Heyms Lyrik hat Silvio Vietta jene mythischen Mächte als Allegorien für Verdinglichungseffekte gedeutet, die von den ungesteuerten Prozessen des beschleunigten Großstadtwachstums im Deutschland der 1910er Jahre ausgehen. ${ }^{23}$ Der gelebte Raum der Großstadt wandelt sich unter den Bedingungen erhöhter Mobilität, des Wachstums unwirtlicher, industriell

21 Am 17.07.1905 notiert Heym in seinem Tagebuch, dass Doves Caracosa "garnicht so bekannt und beliebt" sei (Georg Heym. Bd. 3, S. 33).

22 Georg Heym. Bd. 2, S. 159.

23 Vgl. Silvio Vietta u. Hans-Georg Kemper: Expressionismus. München 1975, S. 49-61. 
geprägter Viertel und der Zunahme der Bevölkerungsdichte auf Weisen, welche Ansprüche auf selbstbestimmtes Handeln durchkreuzen, und die Häufung schockhafter sinnlicher Reize als Folge neuer Transporttechniken, der Beleuchtung, der Reklame und der massenmedialen Kommunikation überlastet das wahrnehmende Bewusstsein auch in Bezirken des Amusements. Die Bilder unausweichlicher Nähe zu abstoßend depersonalisierten Mitmenschen und die Personifizierung der Dinge in Heyms Groteske sind typisch für die Darstellung der Großstadt im zivilisationskritischen Expressionismus. In seiner nachgelassenen Schrift Eine Fratze (1911) konstatiert Heym einen umfassenden Wertezerfall, dem er den trotzig-heroischen Gestus des Weiterlebens im Namen eines schlechthin „Unbekannten" entgegenhält. ${ }^{24}$ Der Betrunkene drückt zwar keine heroische Haltung aus, doch die ästhetische Negation seines avitalen Daseins stützt sich ebenfalls auf Werte, die in der wissbaren Welt nicht Fuß fassen können. Im Zusammenhang des umfassenden Verlusts positiv bestimmbarer Orientierungen entbehrt das Projekt der getarnten Freisetzung von Vitalität, von dem sich das verfremdete ,Ognibene - Caracosa'-Motiv kritisch distanziert, von vornherein der Grundlage. Heym vertritt - bei aller gelegentlichen Feier der Liebe und des Heroismus - oft einen skeptischen Expressionismus, der den Verlust der Fähigkeit zur Selbstbestimmung durch anonyme Zwänge, die aus ungesteuerten Folgen des Denkens und Handelns in der modernen Zivilisation resultieren, als Entfremdung von einer verborgenen metaphysischen Substanz des Bewusstseins darstellt, ${ }^{25}$ und was verborgen bleibt, entzieht sich auch der verdeckten Realisierung.

\section{Bagrow}

In Heyms Frühwerk finden sich sowohl Verschwörer, die - bei allem möglichen Erfolg - unter einem unerfüllten Leben leiden, als auch enthusiastische Revolteure, die ihre anti-vitalen Gegner indessen nicht bezwingen. Zwischen unerfüllter Tarnung und offenem Aufruhr bietet sich kaum Spielraum für eine verdeckte Freisetzung von Vitalität. Heyms spätes Prosastück Bagrow, dessen Handschrift darauf schließen lässt, dass es sich „um den ersten Teil einer längeren Erzählung" handelt, ${ }^{26}$ die er nicht mehr ausgeführt hatte, kompliziert jenes Muster: Der Attentäter Bagrow, der unter dem Entzug von Lebensfülle leidet, versucht vergeblich, am Habitus eines Mitverschwörers die Spuren einer gut getarnten Genialität abzulesen. Die Erzählung verarbeitet einen aktuellen zeitgeschichtlichen Stoff:

Am 14. September 1911 wurde der russische Ministerpräsident Stolypin, als er mit dem Zaren einer Opernaufführung in Kiew beiwohnte, durch einen Pistolenschuß schwer verletzt und starb wenige Tage darauf. Der Attentäter, ein Rechtsanwaltsgehilfe namens Bagrow, wurde am 25. September hingerichtet. Über die Gründe des Attentats gibt es keine zuverlässigen Nachrichten. Die Ochrana (Geheimpolizei), in deren Dienst Bagrow seit 1906 gestanden haben soll, hat sich offenbar darum bemüht, die tatsächlichen Zusammenhänge nicht an die Öffentlichkeit dringen zu lassen. Heyms Prosastück [...] entstand 1911 und geht auf Presseberichte zurück. ${ }^{27}$

Kuljabko, der Leiter der Ochrana in Kiew, hatte laut Pressebericht des Berliner Tageblatts vom 25. September ausgesagt, Bagrow habe zwei Revolutionären, die

24 Georg Heym. Bd. 2, S. 173f., hier S. 174.

25 Krause: Expressionismus, S. 107-109.

26 Seiler: Die historischen Dichtungen Georg Heyms, S. 303.

27 Ebd. 
Stolypins Äußeres studieren wollten, um ein Attentat vorzubereiten, mit Hilfe der Ochrana Zugang zur Oper verschafft; ${ }^{28}$ tatsächlich war der Anschlag aber wohl bereits für die Aufführung geplant.

Heyms Prosastück handelt vom Vorabend des Attentats, an dem der mutmaßliche Lockspitzel Murawjew (bei Heym ,Murawiew` geschrieben) im Büro Kuljabkos stirbt; die Presseberichte über den Vorfall können nicht klären, ob er Selbstmord beging, um sich seinem Auftrag zu entziehen, den Anschlag auf Stolypin auszuführen, oder ob er von der Polizei entwaffnet und erschossen wurde, um das Attentat zu verhindern. Heyms Murawiew folgt Bagrow und einem Mitverschwörer namens Robotnikow, die ihn als designierten Attentäter Stolypins an die Ochrana zu verraten scheinen, in Kuljabkos Büro, und um seiner Verhaftung zu entgehen, erschießt er sich dort. Die drei Agenten haben diese Szene einschließlich der Selbsttötung jedoch einvernehmlich inszeniert, damit die Ochrana glaubt, das geplante Attentat sei nun geplatzt, und ihr Leiter Kuljabko wird in seiner Illusion bestärkt, Robotnikow werde dessen Ausführung übernehmen. Der karrieristische Polizeichef will sich mit der Verhaftung des Robotnikow zu einem späteren Zeitpunkt die Beförderung zum Generalmajor verdienen und wird von Bagrow doppelt getäuscht; während Kuljabko meint, Bagrow wolle mit dem Verrat an Murawiew und Robotnikow in den Rang eines Polizeidirektors aufsteigen, plant Bagrow von Anfang an, Stolypin zu töten, und zu diesem Zweck will er die Kiewer Ochrana mitsamt ihrem Leiter mit einem Scheinverrat in Sicherheit wiegen. Die List gelingt: Kuljabko, der Bagrow als Mitstreiter auf seiner Seite wähnt, gibt ihm Eintrittskarten für die Opernaufführung und legt so unfreiwillig den Grundstein für Stolypins Ermordung.

Bagrow, der das Ideal eines genialen revolutionären Heldentums hegt, macht die ständige Verstellung im Alltag zu schaffen: „Er wollte sich nicht verraten. Man mußte vorsichtig sein. So sehr war er nicht mehr Herr seiner selbst. Mein Gott, er zitterte immer". ${ }^{29}$ Als ihm in seinem Büro ein Telegramm zugestellt wird, von dem er zu Recht glaubt, dass es den verschlüsselten Auftrag zum Mord an Stolypin enthält, bereitet es ihm Mühe, seinen inneren Zustand angesichts der Gewissheit, bald einen Menschen zu töten und anschließend hingerichtet zu werden, vor den Mitarbeitern zu verbergen: „Er wußte nicht recht, was er sagte. Er achtete nur auf den Klang seiner Stimme, ob er natürlich schien, oder ob er Verdacht erregen mußte."30 Murawiew ist als Kontrastfigur zu Bagrow angelegt; ihm scheint die Verstellung mühelos zu gelingen, weil er so seine maskuline Natur verwirklicht:

\footnotetext{
Bagrow beneidete ihn. Ach, bei dem war alles so einfach. Jetzt ging er sterben, als wenn das so gar nichts wär. So einfach, aufstehen, seinen Revolver nehmen, sich von Kuljabko auf den Kopf zu sagen lassen: „Sie sind der Mörder, der morgen das Attentat ausführen soll“, und sich dann eine Kugel vor den Kopf schießen und umfallen wie ein Baum.

So würde der da es machen. Ja, das war noch ein Mann. Wie er da stand, und sich von Robotnikow verabschiedete, der seine Hände nicht loslassen wollte. „Na, wenn der da umfällt, Bruder, dann kommst du ran. ${ }^{\text {31 }}$
}

Im Unterschied zu Bagrow, der mit erratischen Gesten bei Mitarbeitern in seinem Büro den Eindruck erweckte, verrückt zu werden, verrät Murawiew keine Unsicherheit: „Bagrow beobachtete Murawiews Gesichtszüge heimlich. Aber der hatte seine

28 Ebd., S. 305-306

29 Georg Heym: Bagrow. In: Georg Heym. Bd. 2, S. 151-156, hier S. 151.

30 Ebd., S. 152.

31 Ebd., S. $153 f$. 
Augen überall. Man konnte ihm wirklich nichts ansehen. Donnerwetter." Dennoch kommen Bagrow Zweifel an der Authentizität der darin scheinbar zum Ausdruck kommenden heroischen Gesinnung: „Oder ob er posierte? Dann war er einer der glänzendsten Schauspieler."32

Und er dachte: „Es kommt für die Umwelt auf dasselbe heraus, ob das was man tut, wahr ist oder nur wahr scheint; der Eindruck entscheidet."

Etwas an dieser Formulierung paßte inm nicht. Er stieß sie wieder um. Er quälte sich mit einer neuen ab. Schließlich wollte er diesen Gedanken ganz von sich stoßen. Aber er kam immer wieder. Ach mein Gott, ich werde verrückt, wiederholte er sich. ${ }^{33}$

Bagrow versucht, den Zweifel am Augenschein im Vertrauen auf momentane sinnliche Gewissheiten zu neutralisieren, kann ihn aber weder zurückweisen noch bestätigen. Als inm Murawiew vor Kuljabkos Büro eine Brosche mit der Bitte übergibt, sie als „kleines Andenken“ seiner Schwester zu geben, denkt er: „Er posiert also doch." ${ }^{34}$ Die zeitliche Abstimmung und das Untertriebene dieser Geste scheinen auf eine Inszenierung von Größe zu verweisen, mit der die Angst vor dem gewissen Tod überspielt werden soll:

[...] Da schaute eben der Eselsfuß unter der Löwenhaut vor.

Aber wenn er nun doch ein Kind war, ein Genie, ein Heros. Ach vielleicht war er beides, eine seltsame Mischung, von einem Feigling, der sich in der Rolle eines Helden gefiel, und von einem wirklichen Helden. ${ }^{35}$

Auch Bagrows Versuch, das Nacheinander überzeugender und zweifelhafter Eindrücke als stimmigen Ausdruck ambivalenter Charakterzüge zu denken, bleibt bloße Spekulation. Eine Lebensführung, die sich der Verstellung widmet, um unter dem Opfer des eigenen Lebens den Träger einer entfremdenden Macht zu töten, gibt nach außen hin nicht zu erkennen, ob sie sich aus einem naturwüchsig belebten Zustand speist oder ihr Heldentum lediglich inszeniert. Der Versuch, die Authentizität verschwörerischer Handlungen am Habitus eines Akteurs abzulesen oder mit dem eigenen Habitus zu bekräftigen, muss scheitern: „Ob sie ihm mißtrauten. Er mußte sich reinwaschen, um Gottes willen nur nicht dieses Mißtrauen." ${ }^{36}$ Bagrow scheint wegen seines früheren Verrats an Mitstreitern oder angesichts seiner skeptischen Erwägung der Unmöglichkeit reiner Motive - Grund zu haben, mögliche Zweifel an seinen revolutionären Zielen abzuwenden. Kuljabko hingegen meint, der getarnten Persönlichkeit Bagrows unverzerrt bei der Arbeit zusehen zu können, täuscht sich aber - und stinkt dabei "nach Zwiebel“; ${ }^{37}$ mit dem Geruchsmotiv wird eben die Figur, die meint, den inszenierten Augenschein zu durchschauen, als besonders abstoßend dargestellt. Die Erzählung problematisiert den Versuch, sich aus der Perspektive einer umfassend verunsicherten Maskierung einer ungekränkten Vitalität hinter den getarnten Handlungen eines Mitverschwörers zu vergewissern, und auf diese Weise nimmt Heym zu aktuellen Diskussionen über Bagrow Stellung. Heym führt die undurchsichtige Rolle, die der historische Attentäter als Verschwörer und agent provocateur

32 Ebd., S. 154.

33 Ebd.

34 Ebd.

35 Ebd., S. 155.

36 Ebd., S. 154.

37 Ebd., S. 155. 
gespielt hatte, auf revolutionäre Absichten zurück, die sich gegen lähmende Selbstzweifel behaupten müssen.

Stolypins Agrarreformen durchkreuzten Interessen der Sozialrevolutionäre, weil sie eine soziale Spaltung bäuerlicher Schichten begünstigten, und wurden von einer Friedenspolitik flankiert, die Teilen des militärischen Establishments zuwider war. Vor dem Hintergrund dieser Spannungen ist über eine Begünstigung oder Initiierung des Attentats durch die Geheimpolizei spekuliert worden. ${ }^{38}$ In der Zeitschrift Jugend erschien nach dem Anschlag die Karikatur Russisches System von Friedrich Heubner, in der ein russischer Polizist einem Revolutionär zuruft: „Was, gestohlen hast und umgebracht auch schon! Du bist mein Mann! Du kommst sofort unter die Geheimpolizei und zwar direkt zum Schutze des Zaren!"39 Als mögliche Quelle Heyms erwägt Bernd W. Seiler einen Artikel von Franz Pfemfert in der Zeitschrift Die Aktion vom 25. September 1911. ${ }^{40}$ Dort wird Bagrow als Lockspitzel im Dienst der Ochrana dargestellt, der den Vorwand zu verschärften Maßnahmen gegen Revolutionäre liefern sollte; der letztlich tödliche Schuss auf Stolypin sei von der Polizei indessen nicht geplant gewesen und verdanke sich dem Übereifer des Agenten. In Heyms Erzählung entspringt die Tötung jedoch keinem Fehlgriff aus Übereifer, sondern ist von vornherein geplant.

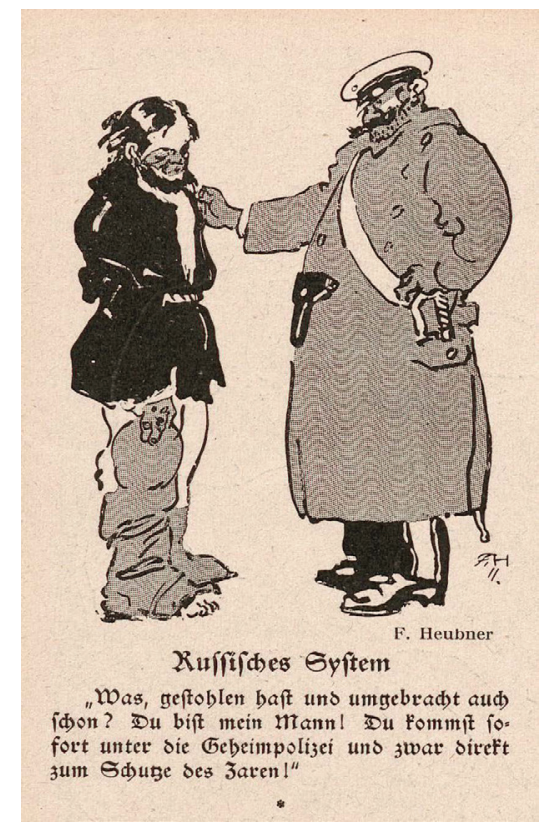

38 Auch die jüngere historische Forschung hat einschlägige Mehrdeutigkeiten nicht auflösen können: „In truth, we do not know what" Bagrow "was planning to do, and it is conceivable that he himself did not know" (Abraham Ascher: P. A. Stolypin: The Search for Stability in Late Imperial Russia. Stanford 2001, S. 378; siehe ebd., S. 161, zu Lenins Opposition gegen Stolypins Reformen, und ebd., S. 384-386, zur Hypothese, das Attentat sei ein Anschlag der politischen Rechten gewesen; Ascher kann entsprechende Verschwörungstheorien nicht bestätigen).

39 In: Jugend. 1911 (Jg. 16), Nr. 40, S. 1080. Siehe https://digi.ub.uni-heidelberg.de/diglit/jugend1911_2/0391, Zugriff 29.07.2020.

40 Vgl. Seiler: Die historischen Dichtungen Georg Heyms, S. 304f. 
Heyms revolutionär ausgerichtete Bagrow-Figur passt eher zur Sicht des Anarchisten Erich Mühsam, der in diesem Attentat ein „Signal zu neuem Anmarsch auf dem Wege der Revolution" erblickte. ${ }^{41}$ Bei allem Verrat, den er in seiner Verbindung mit der Ochrana an Einzelnen begangen haben mag, habe Bagrow mit dem Schuss auf Stolypin letztlich eine reine Gesinnung unter Beweis gestellt:

\begin{abstract}
Dass er mit der verworfenen Horde, die in Russland unter dem Namen politische Polizei über die Gesinnungsreinheit der Menschen wacht, Verbindung hatte, scheint ja der Fall zu sein. Wie weit er die Verräterei gegen seine Freunde trieb, lässt sich vorläufig nicht übersehen. Da er eben sein Leben für die Sache der Freiheit gelassen hat, mögen psychologische Erörterungen beiseite bleiben. Sicher ist, dass das schwere Werk, das er auf sich nahm und mit erstaunlichem Mut ausführte, nur in heiliger Begeisterung für eine Idee reifen konnte. Bagrow hat sich mit seiner Tat auf das Vernehmlichste aus der Gemeinschaft des Lockspitzel-Gesindels, auf dem die Sicherheit des russischen Staates beruht, losgesagt, er hat, was er vorher gefehlt haben mag, mit dem Tode bezahlt, und darum soll sein Name im Gedächtnis freiheitlicher Menschen in Ehren fortleben. ${ }^{42}$
\end{abstract}

Es gibt keinen Beleg für Heyms Lektüre von Mühsams Aufsatz, und über persönliche Kontakte der beiden lässt sich nur spekulieren. Emmy Ball-Hennings zufolge besuchte Heym öfter das Münchner Lokal Simplicissimus, das unter anderem von Erich Mühsam frequentiert wurde; ${ }^{43}$ am 16. und 17. November 1911 und einige Tage danach war Heym in München, ${ }^{44}$ und auch zur Jahreswende war er dort. ${ }^{45}$ Mitte November 1911 war Mühsam aber in Berlin; die Silvesternacht verbrachte er zwar im Simplicissimus, wo er und Heym sich hätten treffen können, ${ }^{46}$ doch dass Heym noch 1912 am Bagrow-Stoff gearbeitet hätte, ist nicht verbürgt. Jedenfalls berühren sich Heym und Mühsam in der Bereitschaft zur spekulativen Einfühlung in einen Attentäter, dessen sozialrevolutionäre Zielsetzung - anders als bei Pfemfert - außer Zweifel steht; Heym stellt die psychologische Dimension, die bei Mühsam ausgeklammert wird, indessen in den Vordergrund, und er problematisiert dabei den Glauben an eine heilige Begeisterung des Attentäters.

Heyms Notizen zur unausgeführten Fortsetzung der Erzählung heben Bagrows starke Zweifel hervor, ob er Stolypin töten darf und soll. Heyms Bagrow ist kein begeistert tatkräftiger Mensch, dessen vitale Energien nur darauf warteten, sich im rechten Augenblick in heroischer Größe zeigen zu können; vielmehr sieht er sich schon am Vorabend des Attentats als Gefangenen und wird sich des bevorstehenden Verlusts seiner Freiheit bewusst:

Alles was er zum letzten Mal tat. [...] Abschied von seiner Freundin. Will ihr was mitbringen. Kehrt wieder um. Geht allein durch die Straßen. Sein letztes Glück dieser einsame Spaziergang. [...] Aber wie kann ich meinen Schmerz an anderen auslassen. Ja hätte ich eine große Seele. Aber ich habe keine große Seele, mein Gott, ich bin ein armer Russe, ein armer Leidender, etwas Getriebenes [... . ${ }^{47}$

41 Erich Mühsam: „Bemerkungen“. In Kain. Zeitschrift für Menschlichkeit. 1911 (Jg. 1), Nr. 7, S. 110-112, hier S. 110.

42 Ebd., S. $110 f$.

43 Aus einem Brief vom 6.2.1946 von Emmy Ball-Hennings. In: Karl Ludwig Schneider u. Gerhard Burckhardt: Georg Heym. Dokumente zu seinem Leben und Werk. München 1968, S. 89-90, hier 89; siehe ebd., S. 555, zu den Besuchern des Kabaretts.

44 Vgl. Schneider (Hrsg.): Am Ufer des blauen Tags, S. 183 u. 192.

45 Vgl. ebd., S. 191 u. 194.

46 Vgl. Volker Griese: Erich Mühsam Chronik: Leben, Werk, Wirkung. Norderstedt 2019, S. 145 u. 150; Schneider (Hrsg.): Am Ufer des blauen Tags, S. 191.

47 Seiler: Die historischen Dichtungen Georg Heyms, S. 304. 
Als erfolgreicher Attentäter wird Bagrow seine Zweifel an seinem Plan überwinden, doch in den Notizen deutet nichts darauf hin, dass er mit der Tat über seine Seelenschwäche hinauswächst. Einerseits stellt sich Heym gegen die Ansicht, Bagrow sei ein bloßer Spitzel gewesen, dem nur ein Fehler unterlaufen ist; andererseits zeigt Heym einen Revolutionär, der unter unabweisbaren und unüberwindlichen Selbstzweifeln leidet.

Martens zufolge interessiert sich Heym für Aufruhr und Revolution als Beispiele des spontanen Aufbruchs zu einem "bewegten, tatvollen Dasein", dessen gesteigertes Lebensgefühl als Offenbarung einer sinnstiftenden Ursprungsmacht gelte. Dieser Zugang zur Revolutionsthematik sei typisch für den Expressionismus: „[...] immer wieder steht die Loslösung von einer lebenhemmenden Umwelt oder deren Vernichtung im Vordergrund, während das Ziel dieses Aufbruchs nur in verschwommenen Umrissen oder überhaupt nicht in Erscheinung tritt". ${ }^{48}$ Bei Heym zeigt sich schon 1909 ein vitalistisches Revolutionsverständnis; so klagt er über „Faule Zeiten, wo der bleierne Frieden / Alles Schöne schon mit Staub umzogen, / Wo erstickt sind groBer Taten Wogen. / Barrikaden, wird man euch noch schmieden?" Wolfgang Rothe führt diese Zeilen als Beleg für den Wunsch der Expressionisten nach dem Aufbruch zu einem befreiten Dasein durch eine "humane und menschliebende Tat" an. ${ }^{49}$ Noch am Tag nach dem Attentat auf Stolypin notiert Heym in seinem Tagebuch den Wunsch nach enthusiastischer Hingabe an eine Revolution, „wo ich mit Anstand hätte mein Leben lassen können". ${ }^{50}$ Mit Bagrow setzt Heym seine Arbeit an sozialrevolutionären Stoffen fort, ohne die aufrührerische Tat zum Aufbruch zu stilisieren. Freilich wissen wir nicht, ob sich Heyms Bagrow bei einer weiteren Ausarbeitung im Augenblick des Attentats im Bann der Eruption von Vitalität vielleicht doch noch als bewegter Held entpuppt hätte, oder ob es nur zu einer leidenschaftslosen Hinrichtung gereicht hätte. ${ }^{51}$ Der Versuch, an einem Mitverschwörer, der aus Überzeugung für die Freiheit kämpft, die verdeckte Freisetzung von Vitalität abzulesen, wird aus der Perspektive einer umfassenden Verunsicherung über den eigenen Selbstwert jedenfalls grundlegend problematisiert.

\section{Getarnte Vitalität: vom scheiternden Projekt zur vergeblichen Illusion}

In der nachgelassenen Erzählung Die Novella der Liebe (1907) hatte Heym eine Liebe gefeiert, deren sexuelle Energien bei heimlichen, anfangs noch keuschen Zusammenkünften schließlich zum erfüllenden Durchbruch kommen. Die mit List und Betrug in einer verständnislosen religiösen Mitwelt behauptete Liebe zieht die Geburt eines Kindes nach sich, an der die Mutter, die das Neugeborene aus Scham und Verzweiflung tötet, stirbt; der entlarvte Vater des Kindes wird schließlich von einer

48 Martens: Vitalismus und Expressionismus, S. 250.

49 Wolfgang Rothe: Tänzer und Täter. Gestalten des Expressionismus. Frankfurt am Main 1979, S. 130 u. 131.

50 Georg Heym. Bd. 3, S. 164; vgl. dazu auch auch Heyms Gedicht Alle Alchimisten brennen voll Rauch ... In: Georg Heym. Dichtungen und Schriften. Bd. 1: Lyrik. Hrsg. v. Karl Ludwig Schneider. Hamburg u. München 1964, S. 354f., hier S. 354.

51 In einer anderen Prosaskizze scheint eine "Krisis" der "Mutlosigkeit" zumindest zeitweilig abrupt überwunden zu werden (Heym: Skizzen, S. 159f., hier 160); allerdings geht dieser Wandel mit dem Wechsel der Erzählperspektive von der dritten zur ersten Person einher. 
erregten Menge von einem Turm geworfen, vergewissert sich aber noch im Fallen der Macht der "großen - herzbezwingenden und vernichtenden" Liebe. ${ }^{52}$ Seine in getarnter Form wenigstens zeitweilig freigesetzte Vitalität wird am Ende der Erzählung noch im Augenblick des Todes uneingeschränkt bejaht. Gewiss, noch im späten Werk feiert Heym die belebte Erfüllung in Liebesverbindungen am Rande oder außerhalb der menschlichen Gesellschaft, ${ }^{53}$ die aber keine Verstellung mehr erfordern.

Ab 1911 wird bereits die Idee der getarnten Freisetzung von Vitalität zum Problem. Der Betrunkene in der grotesken Prosaskizze ist so orientierungslos, dass für die poetische Bejahung des verdeckten Festhaltens an einem gescheiterten Liebeswunsch kein Raum mehr bleibt, und Bagrows verkappter Freiheitskampf verfehlt die Lebensfülle, in deren Namen er gern kämpfen würde, so umfassend, dass er unfähig ist, seine Mitwelt im Lichte jener Idee zu deuten. Als Bagrow heimlich die Hand des Murawiew, der auf einem Lumpenwagen abtransportiert wird, wie zum Abschied noch einmal ergreift, fühlt er nur die Starre des Toten. ${ }^{54}$ In seinem Dramenfragment Lucius Sergius Catilina hatte Heym schon 1908 einen Verschwörer dargestellt, der unter dem Entzug von Vitalität leidet:

Ach genießen. Genießen.

Einmal eine reine Freude fühlen dürfen, einmal wie ein Knabe sein, da das Wunder zu erleben, einmal eine Nacht lang vor Glück nicht schlafen können. Übersättigter Verstand, der immer das schale Ende aller Dinge sieht. 55

Catilina leidet unter dem ernüchternden Bewusstsein der Vergänglichkeit des Ruhms, den er anstrebt, sowie am Missverhältnis seiner Ziele und Mittel: „Und wieviel Qualen müssen unsern Ruhm aufwiegen." 56 Auf ähnliche Weise leidet auch der Attentäter Gylippos in Heyms Drama Der Feldzug nach Sizilien (1910) an einem Mangel an Leidenschaft: „Ich habe solchen Hunger nicht nach ihr, / Wie oft von dieser Stunde ich gemeint. / [...] / Ich will ihn töten, denn umsonst nicht will ich / Mein Leben dieser Tat geopfert haben." Er erkennt auch den Antagonismus von Tarnung und Vitalisierung: „Ich will die Stunde nicht verziehen lassen, / Dafür ich manches Jahr der Erde Freude, / Wein, Weib, Musik und Freund für nichts geachtet / Und gern verschmäht."57 Bagrow leidet indessen nicht an der Entkräftung seiner Wünsche oder dem Aufschub von Erfüllung: nicht Velleität und Askese sind sein Problem, sondern der Zwang zur umfassenden Selbstkontrolle als Folge seiner Unsicherheit über die Wirkung seines verstellten Handelns - und der daraus erwachsende Entzug von Spielräumen erfüllender Spontaneität. Das Thema dekadenter Willensschwäche weicht der expressionistischen Diagnose des Zerfalls eines kohärenten Bewusstseins unter unpersönlichen Zwängen, die sich gegenüber dem Willen der Beteiligten verselbständigt haben und diesen durchkreuzen. Ähnlich geht es dem Betrunkenen in Heyms grotesker Prosaskizze: Das Stadtleben wird von anonymen Prozessen der sozialen Entfremdung heimgesucht, die so umfassend ist, dass es keinen Sinn mehr

52 Georg Heym: Die Novella der Liebe. In: Georg Heym. Bd. 2, S. 100-105, hier 105.

53 Georg Heym: Der Höhenmesser zeigte... u. Das Geheimnis der Liebe. In: Georg Heym. Bd. 2, S. $145 f$. u. S. $146-148$.

54 Heym: Bagrow, S. 156.

55 Heym: Lucius Sergius Catalina. In: Georg Heym. Bd. 2, S. 655-666, hier S. 661.

56 Ebd.

57 Georg Heym: Der Feldzug nach Sizilien. In: Georg Heym. Bd. 2, S. 185-334, hier S. 331. 
gibt, der sich - wenn auch vergeblich - ins verdeckte Handeln retten ließe. In den hier besprochenen Prosatexten von 1911 versucht Heym, ein Thema, das ihn schon in seiner frühen Schaffensphase beschäftigt hat, in den Horizont einer expressionistischen Zivilisationskritik einzuholen, was ihm im Bagrow-Fragment gelungen ist: Der Versuch, sich einer sinnentleerten sozialen Welt der normativ entgrenzten Konkurrenz um Macht dem Schein nach anzupassen, um eine Gelegenheit zu finden, mit der Tötung ihrer Machtträger ihre Grundlagen zu zerstören, erzwingt einen kontrollierten Selbstbezug, der den Zugang zu eben derjenigen ungekränkten Subjektivität, die das verstellte Handeln befreien will, verlegt. Aus der Perspektive einer umfassend funktionalisierten Praxis, die sich nur noch am Tauschwert der gelungenen Anpassung an fremdbestimmte Zwänge ausrichtet, gibt es keine ungezwungene Subjektivität, die sich in offener oder verdeckter Form im Handeln zeigen könnte; wird die Gültigkeit einer solchen Praxis davon abhängig gemacht, dass sie das zugrunde liegende Ethos zumindest intrasubjektiv glaubwürdig zum Ausdruck bringt, erliegt der Akteur einem unbegriffenen Zwang zur grundsätzlich scheiternden Selbstvergewisserung. ${ }^{58}$ Martens und Rothe betonen zu Recht, dass eine gewichtige Reihe von Texten des Expressionismus mit messianischem Pathos das revoltierende oder revolutionäre Handeln zur Manifestation der erlösenden Substanz menschlicher Freiheit stilisiert, und auch Heym neigt immer wieder einmal zu dieser Position. Bagrow bringt diesen Messianismus aus der Sicht eines skeptisch ausgerichteten Expressionismus jedoch zum Scheitern, und als Beitrag zur Kritik selbstzerstörerischer Formen des politischen Widerstands gegen Fremdbestimmung ist dieser Text bislang zu Unrecht vernachlässigt worden. Man braucht dem Vitalismus, der diese Kritik leitet, nicht zuzustimmen, um die genaue Auslotung seiner Aporien im Kontext einer Verschwörung, die sämtliche Kräfte des Akteurs absorbiert, aus dem historischen Abstand heraus kritisch zu würdigen.

58 Zur Kritik des Expressionismus an zeitgenössischen Spielarten fremdbestimmter Funktionalisierung siehe Krause: Expressionismus, S. 88-106. 